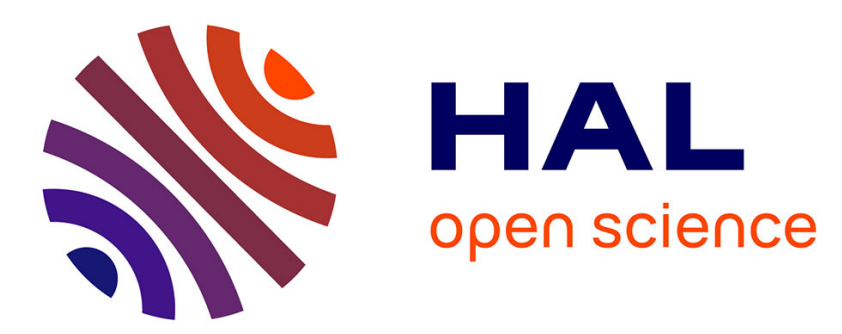

\title{
Electronic and spin delocalization in a switchable trinuclear triphenylene trisemiquinone bridged $\mathrm{Ni3}$ complex
}

Yiting Wang, François Lambert, Eric Rivière, Régis Guillot, Christian Herrero, Antoine Tissot, Zakaria Halime, Talal Mallah

\section{To cite this version:}

Yiting Wang, François Lambert, Eric Rivière, Régis Guillot, Christian Herrero, et al.. Electronic and spin delocalization in a switchable trinuclear triphenylene trisemiquinone bridged $\mathrm{Ni3}$ complex. Chemical Communications, 2019, 55 (82), pp.12336-12339. 10.1039/C9CC05183J . hal-02322279

\section{HAL Id: hal-02322279 \\ https://hal.science/hal-02322279}

Submitted on 10 Nov 2020

HAL is a multi-disciplinary open access archive for the deposit and dissemination of scientific research documents, whether they are published or not. The documents may come from teaching and research institutions in France or abroad, or from public or private research centers.
L'archive ouverte pluridisciplinaire HAL, est destinée au dépôt et à la diffusion de documents scientifiques de niveau recherche, publiés ou non, émanant des établissements d'enseignement et de recherche français ou étrangers, des laboratoires publics ou privés. 


\section{ChemComm}

Electronic and spin delocalization in a switchable trinuclear triphenylene trisemiquinone bridged $\mathrm{Ni3}$ complex

\begin{tabular}{|r|l|}
\hline Journal: & ChemComm \\
\hline Manuscript ID & CC-COM-07-2019-005183.R1 \\
\hline Article Type: & Communication \\
\hline & \\
\end{tabular}

SCHOLARONE ${ }^{\text {m }}$

Manuscripts 


\section{Chemical Communications Guidelines for reviewers}

ChemComm is a forum for urgent high quality communications from across the chemical sciences.

Communications in ChemComm should be preliminary accounts of original and urgent work of significance to a general chemistry audience. The 2017 Impact Factor for ChemComm is $\mathbf{6 . 2 9 0}$.

Only work within the top $25 \%$ of the field in terms of quality and interest should be recommended for publication. Acceptance should only be recommended if the content is of such urgency and significant general interest that rapid publication will be advantageous to the progress of chemical research.

\section{Routine and incremental work - however competently researched and reported - should not be recommended for publication.}

\section{Articles which rely excessively on supplementary information should not be recommended for publication.}

Thank you very much for your assistance in evaluating this manuscript.

\section{General Guidance}

Reviewers have the responsibility to treat the manuscript as confidential. Please be aware of our Ethica Luidelines, which contain full information on the responsibilities of reviewers and authors, and our Refereeing Procedure and Policy.

\section{Supporting information and characterisation of new compounds}

Experimental information must be provided to enable other researchers to reproduce the work accurately. It is the responsibility of authors to provide fully convincing evidence for the homogeneity, purity and identity of all compounds they claim as new. This evidence is required to establish that the properties and constants reported are those of the compound with the new structure claimed.

Please assess the evidence presented in support of the claims made by the authors and comment on whether adequate supporting information has been provided to address the above. Further details on the requirements for characterisation criteria can be found here.

\section{When preparing your report, please:}

- comment on the originality, significance, impact and scientific reliability of the work;

- state clearly whether you would like to see the article accepted or rejected and give detailed comments (with references, as appropriate) that will both help the Editor to make a decision on the article and the authors to improve it;

- it is the expectation that only work with two strong endorsements will be accepted for publication.

\section{Please inform the Editor if:}

- there is a conflict of interest;

- there is a significant part of the work which you are not able to referee with confidence;

- the work, or a significant part of the work, has previously been published;

- you believe the work, or a significant part of the work, is currently submitted elsewhere;

- the work represents part of an unduly fragmented investigation. 
Manuscript ID: CC-COM-07-2019-005183

TITLE: Electronic and spin delocalization in a switchable trinuclear triphenylene trisemiquinone bridged Ni3 complex

We thank the reviewers for their work and constructive comments that helped to improve the manuscript. We agree with most of the comments of reviewer 1, however there are some points raised with which we do not agree. We answer, below, all the points raised by the reviewers and we correct the manuscript accordingly.

Referee: 1

Comments to the Author

This manuscript by T. Mallah and coworkers reports on electronic and magnetic properties of a trinuclear nickel(II) complex with

hexahydroxytriphenylene. This type of systems is gaining renovated interest in these times due to their possible application as models for spin interactions in 2D electrically conductive MOF (as in the very recent ref. 8) or as possible building blocks of quantum gates which can be perturbed and controlled by electric field. As such, it would be justified to publish it in Chemical Communications. However, in its present version the analysis of the magnetic properties of the system is not very sound, and neglects quite a large part of the literature of the past quarter of century on $\mathrm{Ni}(\mathrm{II})$-semiquinonato systems. The manuscripts thus require major revision before acceptance.

As a first point, I note that authors simply state that the differences in Ni-O and $\mathrm{C}-\mathrm{C}$ distances are consistent with sq character of the bridging ligand, while it is the absolute value of $\mathrm{C}-\mathrm{C}$ and $\mathrm{C}-\mathrm{O}$ distance which unequivocally indicate this, as well known in literature on metal-sq systems (see e.g. Brown, S. N. Inorg. Chem. 2012, 51, 1251-1260, and C. G. Pierpont, Coord. Chem. Rev. 2001, 216-217, 99).

\section{Answers:}

This is not exactly what was stated in the original version. We did not write that it is the difference between the $\mathrm{Ni}-\mathrm{O}$ and $\mathrm{C}-\mathrm{C}$ bond distances that are consistent with the semi-quinone character. The semi-quinone character is clear from the values of the $\mathrm{C}-\mathrm{O}$ and $\mathrm{C}-\mathrm{C}$ bond distances, this is clear but we will insist more on this point by adding a sentence in the revised version and we will add the relevant references in the literature that support this. Our point goes beyond the (obvious) fact that we have semiquinone character for the three groups. We pointed out (and this is clear in the original version) that the DIFFERENCE between the two C-O bond distances around one of the $\mathrm{Ni}$ ions (Ni1) is larger than this same difference around $\mathrm{Ni2}$ and $\mathrm{Ni3}$ and also the DIFFERNCE between the two Ni-O bond distances around Nil is larger than these differences around Ni2 and Ni3 leading to the conclusion that the semiquinone character is more pronounced around Nil (that has a geometry closer to tbp) than around Ni2 and Ni3 (that have a geometry very close to spy). So, the point was to see if, from structural data, it was possible to see if the semiquinone character is more (or less) pronounced around some of the crystallographically different $\mathrm{Ni}$ atoms. And, indeed, this semiquinone character is more pronounced around Nil leading to the conclusion that the electronic density is more localized around Nil (tbp) than around Ni2 and Ni3 (spy). This idea is very important because we have here an example of a conjugated ligand with a trisemiquinone character in trinuclear Ni(II) complex and we have its crystal structure (for the first time), which allows to get insight from the structure on the electron delocalization of the electrons of the three semiquinone species with the respect to the $\mathrm{Ni}$ (II) ions. I see that we summarized too much the idea in the original 
version, so we will add some sentences for the idea to be clear and we will add the relevant references.

From structural data analysis, the ligand is in the tris-sq state, which might lead an uniformed readder to think that a triradical sttate is attained. This is clearly not the case, but a short explanation on this point is on my opinion essential. Indeed, this is of the utmost importance to understand the reason of the use of the specific spin hamiltonian in the analysis of the magnetic data of 1 .

\section{Answer:}

We completely agree with the reviewer that one may assume that we have a triradical, which cannot be the case and, indeed, the idea that two of the electrons pair up must be introduced before the magnetism part. A sentence is added in the revised version just after the structural analysis part

Concerning this point, the number of parameters used for the fit of the magnetic data is far too high (3 different coupling constants + 3 different 9 values for $\mathrm{Ni}(\mathrm{II})$ centers +3 different $\mathrm{D}$ values + TIP) and can be highly correlated: it is not even clear whether they were all left free or not (no error bars are given for the best fit values). This is of particlar relevance since the fit of the magnetic data provide results which are not in line with expectation from simple magnetostructural correlations for $\mathrm{Ni}(I I)-s q$ exchange. In particular, it is well known that for square pyramidal (or distorted octahedral) Ni(II) systems, the interaction with SQ radical is expected to be ferrromagnetic (see eg Inorg. Chem. 2002, 41, 1086-1092 for an example of $\mathrm{Ni}(\mathrm{II}) 3 \mathrm{SQ3}$ complex), while for tbp is antiferromagnetic (see eg Dei, Gatteschi Inorg. Chim. Acta 198-200 813-822).

The corresponding hamiltonian should then feature an isosceles triangular symmetry with J1=J2 ferromagnetic, and J3 antiferromagnetic. Any deviation from such expectation should be critically analyzed and discussed using simple symmetry based arguments. If this is not possible now, then authors should wait to have their theoretical calculations finished and include them (there is plenty of space before reaching the 4 pages limit of ChemComm). This might also explain the huge TIP value used to fit the data, which accounts for 25\% of the magnetic susceptibility at room temperature, and at the moment has no physically sound explanation.

\section{Answer:}

We agree with the reviewer that we had many parameters and that these parameters can be correlated. That is why when performing the fitting procedure, we had to restrict some parameters within a given range justified by their structure and by results from the literature. We made restriction for the $g$ parameters between 2.0 and 2.4 , which is reasonable for $\mathrm{Ni}(\mathrm{II})$ in pentacoordinate environment. We restricted the D values between 10 and 30 for $\mathrm{Ni2}$ and $\mathrm{Ni3}$ (spy) and between -10 and -40 for Ni1 (tbp). We already mentioned this restriction on $D$ values in the original version. Again these ranges are those expected for the two different geometries based on recent reports by us and others on ZFS of pentacoordinate $\mathrm{Ni}$ (II) complexes. Finally, TIP and J values were left free. We carried out extensive fitting exploring the space of phases starting from different $J$ values. We found that there are ranges of the different $J$ values that give reasonable fits of the experimental data BUT with J1 (with the pseudo tbp Nil) antiferromagnetic and relatively large and J2 and J3 that are much weaker and can be ferro-or antiferromagnetic. This is done in the revised version.

The reviewer states that the coupling between the sq and a spyNi complex must be strongly ferromagnetic as for octahedral Ni complexes. While, the coupling between tbpNi and sq must be antiferromagnetic. Our results are in line with the last statement where we indeed find large antiferromagnetic 
coupling between the pseudotbpNi and sq. For the coupling between pseudospyNi and sq, we do not find a lager ferromagnetic coupling as the reviewer thinks it should be, the coupling is rather weak and may be ferroor antiferro.

We looked back in the literature and considered the reference suggested by the reviewer. Actually, not much is known concerning the relation between geometry and exchange coupling in pentacoordinate Ni-sq complexes. There exist three such complexes reported and only two structures. The oldest compound that the reviewer mentioned ( $\mathrm{Ni}(\mathrm{n} 3)$ ( $\mathrm{DTBSQ}$ ) $\left(\mathrm{ClO}_{4}\right)$ by $\mathrm{C}$. Benelli et al. Inorg. Chem. (1990), 29, 3409 and Dei, Gatteschi Inorg. Chim. Acta (1992) 813-822) where $\mathrm{Ni}$ is assumed to be pentacoordinate (and which is probably the case) has no reported structure so one cannot unambiguously know whether the geometry is top or spy or intermediate. Our experience in pentacoordinate $\mathrm{Ni}$ (II) complexes tells us that top Ni(II) complexes are extremely difficult to obtain (see B. Cahier et al. Chem. Eur. J. 2017, 23, 3648 - 3657). Only when bulky ligands where steric hindrance overcome the better electronic stabilization of spy geometry is present, tbp or pseudotop complexes are obtained. Actually, there is a contradiction between the conclusion on the geometry of this compound ( $\mathrm{Ni}(\mathrm{n} 3)$ ( $\mathrm{DTBSQ}$ ) $\left(\mathrm{ClO}_{4}\right)$ ) between the two papers mentioned above; the first paper (C. Benelli et al. Inorg. Chem. (1990), 29, 3409) concludes that the geometry is spy based on EPR, while the second of the same authors (Dei, Gatteschi Inorg. Chim. Acta (1992) 813-822) concludes that the geometry is tbp, also based on EPR. Saying all that, there is an experimental definitive result concerning this compound is that the exchange is strongly antiferromagnetic when Ni(II) is in a pentacoordinate geometry, while it is accepted that for octahedral Nisq complexes, the coupling is rather ferromagnetic and large (relevant references are added in the revised version.

We, therefore, explored the literature and the CCDC base looking for pentaNi-semiquinone complexes with structure reported. We found only two complexes one with a quasi-spy geometry (Addison parameter tau=0.05) that presents, on the basis of solution NMR study (220-290 K) and then confirmed by DET calculation (C. Cox et al., Polyhedron 162, (2019), 165-170), a strong antiferromagnetic exchange coupling. Therefore, the coupling between a spyNi and semiquinone is antiferromagnetic and ferromagnetic as in octahedral $\mathrm{Ni}$ complexes. The other complex we found has a geometry closer to tbp (Addison parameter 0.63 very close to that of Nil 0.65) and where the coupling is also strongly antiferromagnetic. So in conclusion, it appears that for pentacoordinate Ni-sq complexes, the exchange coupling parameter is antiferromagnetic whether the geometry is pseudo square pyramidal or trigonal bipyramidal and can be very strong.

Our results are not in contradiction with those of the literature and the antiferromagnetic coupling between a distorted spyNi and semiquinone was justified by DFT calculations (C. Cox et al., Polyhedron 162, (2019), 165170). However, the comparison between our complex and those of the literature is not straightforward because of the degree of delocalization of the semiquione electron is not the same on the HHTP ring and on a terminal $\mathrm{DBSQ}^{-}$and this may change the magnitude and also the nature of the coupling.

In conclusion to this important (and long I am sorry for that) part, our results are in line with the literature data and the fact that the coupling (antiferromagnetic) is not as large as in the literature is due to the larger delocalization of the single electron of on the large HHTP- ligand. We will correct the text in the revised version in order to express in a clearer manner the ideas and we will site the relevant literature. The only point that is not clear to us is the large TIP value that is perfectly reproducible on three different samples where we took care to have very good data in the high temperature range. We think that this may be due to the presence of close excited states due to metal-to-ligand and more probably to ligand-to-ligand charge transfer that may contribute to 
the temperature independent paramagnetism. Actually, the recently published paper on the Cu(II) trinuclear complex has also large TIP value. This will be mentioned in the revised version.

Finally, calculations are crucial but are out of the scope of a Chem Comm. The problem is that we cannot treat the three Ni-sq species alone, one needs treating the whole molecule, which is long and tedious and probably DFT may not be adapted because different functionals seem to lead to different results (preliminary calculation we performed). We may need to use WF based calculation and probably at the CASPT2 (or NEVPT2 in ORCA) level to be able to get a reliable electronic description together with reliable values of the exchange coupling parameters. We are working on that.

As a final point, the reported EPR spectrum of the doubly reduced species is analyzed by using Ni(II) antiferromagnetically coupled to a SQ: this should then be, according to magnetostructural correlations mentioned above and chemical/statistical arguments the tbp Ni(II) centre; but then the D value should be negative at variance with best simulation parameters.

\section{Answer:}

No, we do not agree. The reviewer is basing his/her argument on the fact that the coupling is antiferromagnetic only when Ni(II) has a geometry close to tbp and not to spy. As we mentioned above, the coupling between $\mathrm{Ni}$ (II) and sq can be strongly antiferromagnetic also when the geometry is spy (C. Cox et al., Polyhedron 162, (2019), 165-170). So our result is in line with the literature and a very strong antiferromagnetic coupling with the spy $\mathrm{Ni}(\mathrm{II})$ is possible; the authors estimate $-1000<\mathrm{J}<-500 \mathrm{~cm}^{-1}$. Finally, the average $g$ value for $\mathrm{Ni}$ obtained from the EPR spectra is 2.15 which is compatible with a geometry close to spy than to tbp (a relevant ref. will be added in the revised manuscript). So our result stating that we have a large antiferromagnetic coupling between a pseudospyNi and semiquinone is perfectly in line with the literature and justified by symmetry arguments (see C. Cox et al., Polyhedron 162, (2019), 165-170)

I also notice that the obtained rhombicity for $g$ value appears to be quite high for $\mathrm{Ni}(\mathrm{II})$. In this respect, I strongly suggest that this spectrum is simply analyzed in term of an anisotropic doublet, and then use of projection coefficient reported in ref. 15 can be used to derive the corresponding anisotropic $g$ values of the single Ni(II) ion.

\section{Answer:}

We agree with the reviewer. This is actually what we have done, but have not explicitly developed it in the paper.

So, we have first simulated the spectrum in term of an anisotropic doublet and extracted the three g components. We will add this spectrum together with the experimental one in the SI of the revised version. Then, we used the projection coeff of ref. 15 (geff $=-g(1 / 2) / 3+4 g(N i) / 3$ ) and computed the values of the $g_{\mathrm{Ni}}$ components assuming that $\mathrm{g}_{\mathrm{rad}}$ isotropic and equal to 2 . We took these values and simulated the experimental spectra with different J/D values (the spectra depend only on J/D as explained in ref.15 of the original ms) with negative and D positive and negative. J was taken negative (antiferromagnetic) because the positive solution leads to a completely different spectrum.

The next step was to adjust the experimental spectrum to the simulation with different $D$ values and it was clear that this was possible only with positive D values and with $|\mathrm{J}| / \mathrm{D}$ around 20. The conclusion is that in the two-reduced species, the single electron on HHTP ${ }^{3-}$ interacts strongly antiferromagnetically with one of the pseudospyNi(II) while it had an antiferromagnetic coupling coupling with the pseudotbpNi(II) in the 
pristine species showing a different spin localization between the two species.

We will revise this part explaining the methodology we used as required by bthe reviewer.

Concerning the high rhombicity of the g value of a pseudospy-Ni(II) mentioned by the reviewer. Actually, even though the geometry is close to spy, it is highly distorted. The $\mathrm{N}_{2} \mathrm{O}_{2}$ plane of the spy is made of electronically different atoms (two $O$ with one negative charge and two neutral nitrogen atoms). The angles around the Ni2 and Ni3 atoms in the plane range from 82 to $95^{\circ}$. In addition, the angle between the normal to the $\mathrm{N}_{2} \mathrm{O}_{2}$ plane and the apical Ni-N direction of the spy is around $10^{\circ}$. The structural (and electronic) parameters shows that the geometry around Ni2 and $\mathrm{Ni3}$ is well distorted from the pure square pyramidal geometry and is responsible of the large rhombicity of the pseudo spy-Ni. We added some sentences in the description of the structure to highlight this distortion from the pure square pyramidal geometry.

Referee: 2

Comments to the Author

Authors reported a trinuclear Ni(II) complex bridged by trisemiquinone. Its structure and magnetic properties were characterized well. In addition, reversible electrochemical response was demonstrated by EPR spectra well. These results would give significant information for designing new switching molecules.

In my opinion, this paper is acceptable for publication in chemical Communications after a minor revision.

Comment

Why are the J, D and $g$ values for Ni2 and Ni3 so different? Are there notable structural difference between Ni2 and Ni3? In addition, the magnetization value at $2 \mathrm{~K}$ and $6 \mathrm{~T}$ (2.4 Bohr Magneton) is larger than the expected value based on the J values (two antiferromagnetic and one ferromagnetic). Although authors said "This solution was discarded." in $\mathrm{SI}$, the simulation results would mislead readers. It needs more careful and adequate explanation.

\section{Answer:}

We agree with the reviewer that putting one of the solutions of the fitting and then discarding it adds confusion so this part will be removed in the revised version. To answer the reviewer question on why this solution was discarded: it is mainly because the D values between of Ni2 and Ni3 are different and this is not physically sound since their geometry is very close. Concerning the difference in the J values, this expresses the delocalization of the single electronic spin over Ni2 and Nil that can be different. Actually, since the molecule does not have a symmetry plane there is no reason that the coupling is the same. 


\section{Electronic and spin delocalization in a switchable trinuclear triphenylene trisemiquinone bridged $\mathrm{Ni}_{3}$ complex}

Received 00th January 20xx, Accepted 00th January 20xx DOI: $10.1039 / x 0 x x 00000 x$
Yiting Wang, ${ }^{a}$ François Lambert, ${ }^{a}$ Eric Rivière, ${ }^{a}$ Régis Guillot, ${ }^{a}$ Christian Herrero, ${ }^{a}$ Antoine Tissot, ${ }^{b}$ Zakaria Halime, ${ }^{\text {T Talal Mallaha* }}$
A trinuclear triphenylene trisemiquinone complex containing paramagnetic $\mathrm{Ni}^{\prime \prime}$ is obtained under ambient condition from the reaction of the deprotonated tricatecholate hexahydroxytriphenylene $\left(\mathrm{H}_{6} \mathrm{HHTP}\right)$ with $\mathrm{Ni}^{\prime \prime}$ capped with a trispyrazolyl borate tridentate ligand. The magnetic and EPR data are consistent with a delocalization of the electronic spin over the three $\mathrm{Ni}^{\prime \prime}$ species. The two-electron reduced complex shows an EPR spectrum corresponding to a $S=1 / 2$ species due a large antiferromagnetic coupling between the radical and only one of the $\mathrm{Ni}$ " ions highlighting the localization of the electronic spin. No EPR signal is observed for the one- and three-electron reduced species consistent with the closed shell of the bridging ligand.

Addressable molecular species, particularly electrically, are relevant to the field of quantum information because they allow, upon the application of an electrical perturbation potential, creating entangled quantum bits and, therefore, building quantum gates. ${ }^{1,2}$ Binuclear paramagnetic metal ions containing species have been recently reported where, upon reduction of the bridging ligand, a large exchange coupling interaction between the metal ions was observed. ${ }^{3,4}$

Highly conjugated triphenylene ligands and particularly the tricatecholate 2,3,6,7,10,11-hexahydroxytriphenylene $\left(\mathrm{H}_{6} \mathrm{HHTP} ; \mathrm{C}_{18} \mathrm{O}_{6} \mathrm{H}_{12}\right)$ are well suited for the design of trinuclear complexes with different ligand centered oxidation states. There are six accessible states that can be accessed by oxidizing HHTP6- $^{6}$ ([cat-cat-cat]; cat = catecholate) to HHTP ([q-q-q]; q = quinone). Trinuclear complexes based on $\mathrm{H}_{6} \mathrm{HHTP}$ were already reported with diamagnetic metal ions ( $\mathrm{Ru}^{\prime \prime}$ and low spin $\mathrm{Co}^{\prime \prime \prime}$ ) and very recently with paramagnetic $\mathrm{Cu}^{11} .{ }^{5-8}$

a. Institut de Chimie Moléculaire et des Matériaux d'Orsay, Université Paris-Sud, CNRS, Université Paris-Saclay, 91405 Orsay cedex, France

b. Institut des Matériaux Poreux de Paris, UMR 8004 CNRS, École Normale Supérieure, Ecole Supérieure de Physique et de Chimie Industrielles de Paris, PSL University, 75005 Paris, France

† e-mail talal.mallah@u-psud.fr.

Electronic Supplementary Information (ESI) available: [details of any supplementary information available should be included here]. See DOI: 10.1039/x0xx00000x
Here, we report the design and the magnetic behavior of a trinuclear $\mathrm{Ni}(\mathrm{II})$ complex $\mathbf{1}$ containing the bridging trisemiquinone derivative $\mathrm{HHTP}^{3-}$. In order to reach the most stable thermodynamic state for the bridging ligand, we carried out the preparation of the complex under aerobic conditions by mixing the mononuclear $\mathrm{Ni}\left[\mathrm{BH}\left(\mathrm{TP}^{\mathrm{Ph}, \mathrm{Ph}}\right)_{3}\right] \mathrm{Cl}$, here $\left[\mathrm{BH}\left(\mathrm{TP}^{\mathrm{Ph}, \mathrm{Ph}}\right)_{3}\right]$ is Tris(3,5-diphenyl-1H-pyrazol-1-yl)(hydrido)borate) ${ }^{9} \quad$ with $\mathrm{H}_{6} \mathrm{HHTP}$ that was deprotonated six times. Elemental analysis is consistent with a neutral complex containing one HHTP and three $\left[\mathrm{Ni}\left[\mathrm{BH}\left(\mathrm{TP}^{\mathrm{Ph}, \mathrm{Ph}}\right)_{3}\right]\right.$ species (see $\mathrm{SI}$ for details). Assuming that $\mathrm{Ni}$ remained in its +ll oxidation state and knowing that the capping ligand is negatively charged, the bridging ligand is, therefore, triply negatively charged ( $\mathrm{HHTP}^{3-}$ ) and is in the [sqsq-sq] state. It has, thus, undergone a three-electron oxidation during the synthetic process.

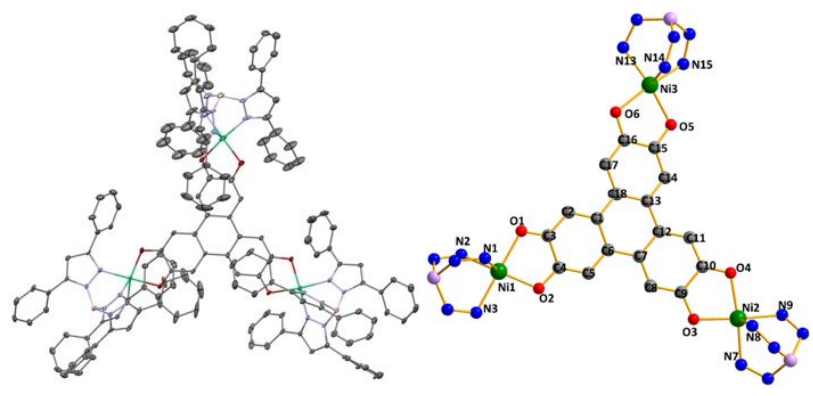

Figure 1. An ORTEP drawing of compound 1. Hydrogen atoms and solvent molecules are omitted for clarity. Only one position of the disordered benzene moieties is shown. Thermal ellipsoids are shown at the $30 \%$ level

The crystal structure of 1 shows the formation of a trinuclear complex (Figure 1 and Figure S1). The three metal ions have a pentacoordinate coordination sphere with two ( $\mathrm{Ni2}$ and $\mathrm{Ni3}$ ) in a distorted square pyramidal (pseudo-spy) geometry, and one (Ni1) is close to trigonal bipyramidal (pseudo-tbp) (Figure S1 and Table 1). The values of the $\mathrm{C}-\mathrm{O}$ and $\mathrm{C}-\mathrm{C}$ bonds around the three $\mathrm{Ni}$ atoms are in line with a semiquinone character (Table S2). ${ }^{10-}$ 12 \{Pierpont, 1981 \#55; \{Lynch, 1981 \#57\}Benelli, 1988 
\#52; Benelli, 1990 \#47; Caneschi, 2002 \#48\} The comparison of the difference between the two $\mathrm{C}-\mathrm{O}$ bond distances in the coordination sphere of each metal ion and then this difference among the three metal ions may give information on how the semiquinone character is more or less pronounced for three metal ions. Furthermore, the comparison of the OC-CO bond lengths of the benzene rings around the three metal ions can also give the same information. Indeed, - tThe differencedifferences between the $\mathrm{C}_{-}-\mathrm{O}$ bond distances is much larger $(0.053 \pm 0.014 \AA)$ around $\mathrm{Ni1}$ (pseudo-that has a-tbp geometry) than around $\mathrm{Ni} 2$ and $\mathrm{Ni} 3$ (less than $0.01 \AA$ ). Also, the $\in C$ bond length is larger $(1.469 \AA)$ around $\mathrm{Ni1}$ than around the other two metal ions $(1.459$ and $1.448 \AA$ ). The difference between the Ni1--O bond distances $(0.080 \pm 0.0107 \AA)$ is larger than between Ni2--O and Ni3-_O $(0.026 \pm 0.013$ Å). These characteristics are consistent with a more pronounced semiquinone character that seems to be localized in the coordination sphere of the pseudo-tbp Ni1 than for the pseudospy $\mathrm{Ni} 2$ and $\mathrm{Ni} 3$. These structural characteristics lead to the conclusion that the semiquinone electronic density in the vicinity of $\mathrm{Ni1}$ is more localized on the OCCO group than in the vicinity of $\mathrm{Ni2}$ and $\mathrm{Ni} 3$ where it is more delocalized towards the central ring of $\mathrm{HHTP}^{3-}$, while around $\mathrm{Ni2}$ and $\mathrm{Ni} 3$, the electron density would be more delocalized. Because the three semiquinone electrons belong to a highly conjugated ligand (HHTP ${ }^{3-}$ ), the three single electrons are expected to undergo a very large antiferromagnetic coupling leading to a doublet ground state $(S=1 / 2)$ well separated from the other excited doublet and quartet states, so that the magnetic properties of the ligand can safely be described as a $S=1 / 2$ below room temperature. This is in contrast to the bis-bidentatebissemiquinone and the tris-bidentate-trissemiquinone ligands reported by Dei and coworkers where conjugation is very weak leading to a weak ferromagnetic coupling between the three semiquinone electrons. ${ }^{13,14}$ 5,6

Cyclic voltammetry study gives information on the relative stability of the different species (Figure 2). The rest potential is at $+0.01 \mathrm{~V}$ vs. SCE (Saturated Calomel Electrode). Scanning towards negative potential leads to three reversible reduction waves at $-0.28,-0.78$ and $-1.17 \mathrm{~V}$ vs. SCE. The pristine species is, therefore, in the $[s q-s q-s q]^{3-}$ state and the three reversible waves correspond formally to the [sq-sq-sq] ${ }^{3-} /[\text { cat-sq-sq] }]^{4-}$, [catsq-sq] $]^{4-} /\left[\right.$ cat-cat-sq] ${ }^{5-}$ and [cat-cat-sq] ${ }^{5-} /[\text { cat-cat-cat] }]^{6-}$ couples. When the potential swiped towards more positive values, only one reversible oxidation wave corresponding to the formation of the $[s q-s q-q]^{2-}$ is observed (Figure S2). The reversibility of the three reduction waves indicates that no major chemical (bond formation or cleavage) or structural (coordination geometry) changes are induced by these redox processes. The comproportionation constants corresponding to the successive reduction processes were found equal to $3.48 \times 10^{8}$ and $4.6 \times 10^{6}$ indicating a less delocalized character of the [cat-cat-sq] ${ }^{5-}$ species. The stable [sq-sq-sq] ${ }^{3-}$ was already observed with Rull metal ions and very recently with $\mathrm{Cu}^{\prime \prime}$ containing complexes, while for the trinuclear Coll' complex, the bridging ligand was found to be in the [cat-cat-sq] ${ }^{5-}$ state.

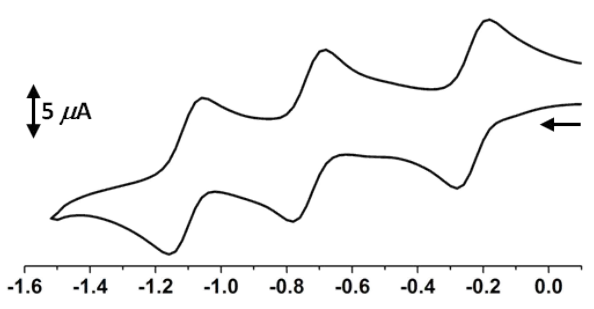

$E$ (V vs. SCE)

Figure 2. Cyclic voltammogram of $1 \mathrm{mM} 1$ in argon-degassed dichloromethane containing $0.1 \mathrm{M}\left[\mathrm{Bu}_{4} \mathrm{~N}\right] \mathrm{PF}_{6}$ at $25{ }^{\circ} \mathrm{C}$ and recorded using a glassy carbon working electrode, a platinum mesh as the counter electrode and a saturated calomel electrode (SCE) as the reference electrode.

Magnetic studies allow getting insight into the interaction between the ligand centered single-electron spin $S=1 / 2$ and the metal centers and may thus give information on the electronic and spin delocalization. The dc magnetic measurements were carried out at $0.1 \mathrm{~T}$ in the $300-2 \mathrm{~K}$ temperature range and at $\mathrm{T}=$ 2, 4, 6 and $8 \mathrm{~K}$ between 0 and $6 \mathrm{~T}$. Upon cooling, $\chi_{M} T$ decreases in a steady manner from 300 to $50 \mathrm{~K}$ and then more abruptly. It reaches a value of $0.9 \mathrm{~cm}^{3} \mathrm{Kmol}^{-1}$ at $2 \mathrm{~K}$, indicating the presence of dominating overall an antiferromagnetic coupling and a nonzero spin ground state (Figure 3 ). The magnetization versus field curves are typical of a paramagnetic ground state with large zero-field splitting (ZFS) because the $M=f(B / T)$ curves are not superimposable (Figure $\mathrm{S} 3$ ). The magnetization value at $2 \mathrm{~K}$ and $6 \mathrm{~T}$ (2.4 Bohr Magneton) is much lower than the expected values for three $\mathrm{Nill}$ complexes and one $\mathrm{S}=1 / 2$ radical even considering large ZFS for $\mathrm{Ni}^{\prime \prime}$. These results indicate the presence of a large antiferromagnetic coupling within the complex. Due to the large separation between the metal ions belonging to different molecules $(13 \AA)$ and the absence of a H-bond network, a magnetic intermolecular interaction at $\mathrm{T}>2 \mathrm{~K}$ can be discarded. Also, since the intramolecular metal-metal distance is large (10 $\AA$ )), the large antiferromagnetic coupling cannot be due to intramolecular direct $\mathrm{Ni}-\mathrm{Ni}$ interaction. In order to fit the magnetic data, we restricted the ZFS values of the different $\mathrm{Ni}^{\prime \prime}$ species to be positive for the pseudo-spy geometry ( $\mathrm{Ni} 2$ and $\mathrm{Ni} 3$; within the $20-30 \mathrm{~cm}^{-1}$ range ) and negative for the pseudo--tbp species ( $\mathrm{Ni1}$; within the $-20--30$ range) as expected based on previous studies. ${ }^{15-18}$ We carriedThe simultaneous fit of $\chi_{M} T$ and magnetization done-using the $\mathrm{PHI}$ software, ${ }^{19}$ withand the following spin Hamiltonian, where we assumed that a central $S$ $=1 / 2$ radical exchange couples with three $\mathrm{S}=1 \mathrm{Nill}$ species:

$H=-J_{1} S_{r a d} \cdot S_{N i 1}-J_{2} S_{r a d} \cdot S_{N i 2}-J_{3} S_{r a d} \cdot S_{N i 3}+D_{1} S_{Z N i 1}^{2}+D_{2} S_{Z N i 2}^{2}+D_{3} S_{Z N i 3}^{2}$

Despite the presence of two Ni(II) with similar pseudo square pyramidal geometry, two different coupling parameters must be considered because the trinuclear complex has no symmetry plane and the central ligand is distorted so there is no physical reason to have exactly the same coupling parameters.

The best fit parameters are leads to the following values: $J_{1}=$ $112 \pm 10.6 \mathrm{~cm}^{-1}, J_{2}=11 \pm 5.4 \mathrm{~cm}^{-1}, J_{3}=-27 \pm 7.6 \mathrm{~cm}^{-1}, D_{1}=-23 \pm$ $\underline{2} .6 \mathrm{~cm}^{-1}, D_{2}=26 \pm \underline{3} .6 \mathrm{~cm}^{-1}$ and $D_{3}=20 \pm \underline{3} .0 \mathrm{~cm}^{-1} ; \mathrm{TIP}=4 \times 10^{-3}$ (see $\mathrm{SI}$ ). The $g$-factors were found equal to $2.14,2.10$ and 2.35 
for $\mathrm{Ni} 1, \mathrm{Ni} 2$ and $\mathrm{Ni3}$ respectively. We performed simulation of the data and found that a range of parameters give reasonable agreement with the experimental ones leading to a range of values for the parameters given by the uncertainties values above. Trials with both $J_{2}$ and $J_{3}$ ferromagnetic or antiferromagnetic may give reasonable agreement with the experimental data but with very different $D_{2}$ and $D_{3}$ which is not physically sound since the geometry around $\mathrm{Ni} 2$ and $\mathrm{Ni} 3$ is very close. While exchange coupling between octahedral $\mathrm{Ni}(\mathrm{II})$ and semiquinone species is established to be strongly ferromagnetic, ${ }^{12}$ the coupling in the case of pentacoordinate ${ }^{20}$ ${ }^{22}$ (for both distorted $\mathrm{spy}^{20}$ and $\mathrm{tbp}^{2119,20}$ geometries) $\mathrm{Ni}(\mathrm{II})$ complexes was found to be strongly antiferromagnetic. ${ }^{20-22}$ Importantly, it has also been shown that the magnitude of the octahedral $\mathrm{Ni}($ II)-semiquinone ferromagnetic coupling decreases when bis-semiquinone or tris-semiquinone ligands bridge two and three $\mathrm{Ni}(\mathrm{II})$ complexes, $^{13,}{ }^{14}$ in comparison to the case of terminal semiquinone where the coupling is very strong. This can be attributed to the delocalization of the semiquinone single electrons on the central bridge. In our case, the central HHTP $^{3-}$ bridging ligand being highly conjugated, a larger delocalization and, hence, a decrease of the magnitude of the exchange coupling is expected in comparison to the cases where semiquinone is terminal, ${ }^{12,}{ }^{20,}{ }^{21}$ therefore, justifying our results. Furthermore, the fact that $J_{2}$ and $J_{3}$ are different is not surprising because the HHTP ${ }^{3-}$ central ligand is distorted and there is no physical reason for the orbital wave function describing the $S=1 / 2$ to have exactly the same weight close to $\mathrm{Ni} 2$ and $\mathrm{Ni3} .{ }^{19}, 20$ These results indicate that the radical undergoes coupling with different magnitudes and nature with the metal ions suggesting a relative delocalization over the ligand $\pi$ skeleton. A knowledge of the nature of the ground state wavefunction is necessary to get insight into the electronic and spin densities distribution and confirm our hypothesis; theoretical calculations are underway to do so but this is out of the scope of this communication.
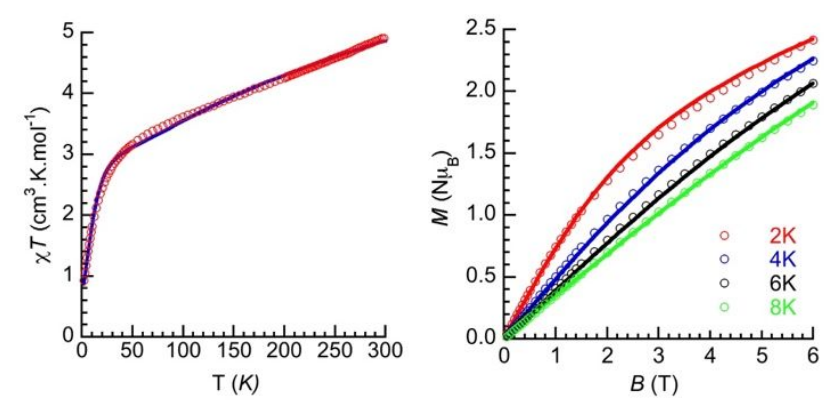

Figure 3. $\chi T=f(T)$ (top) and $M=f(B)$ (bottom) for 1 ; continuous lines correspond to the best fit with parameters in the text

In order to probe the electron delocalization in the reduced species, we recorded the X-band Electron Paramagnetic Resonance (EPR) spectra (perpendicular mode) of the pristine and the three reduced species on solutions obtained by preparative reduction of the sample at $-0.53 \mathrm{~V}\left(\left[(\mathrm{LNi})_{3}\right.\right.$ (cat-sq$\left.\mathrm{sq})]^{-}\right),-0.98 \vee\left(\left[(\mathrm{LNi})_{3}(\text { cat-cat-sq })\right]^{2-}\right)$ and $-1.4 \vee\left(\left[(\mathrm{LNi})_{3}\right.\right.$ (cat-catcat) ${ }^{3-}$ ). The spectra (Figure S4 and Figure 4 ) show that the pristine and the two-electron reduced species present EPR signals, while no signal is observed for the one and the threeelectron reduced species. The absence of an EPR signal is due to the closed shell nature of the bridging ligand and to the fact that isolated $\mathrm{Ni}^{\prime \prime}\left(\mathrm{d}^{8}, S=1\right)$ complexes are EPR silent in X-band perpendicular mode because of their large ZFS in comparison to the photon incident energy $\left(h v=0.3 \mathrm{~cm}^{-1}\right)$. The spectrum of the pristine complex is not intense and observed only below $10 \mathrm{~K}$ (Figure S4); it has several bands indicative of the presence of many low-lying states coming from the interaction between the single electron and more than one $\mathrm{Ni}^{l l}$ confirming in a qualitative manner the magnetic data. Fitting such complex spectrum to obtain more quantitative information is out of scope of this paper. The spectra of the two-electron reduced species is completely different and much simpler; it corresponds to a typical $S=1 / 2$ powder EPR spectra with rhombic $g$ anisotropy. It can be due to the coupling between a single electron localized on the [cat-cat-sq] ${ }^{5-}$ central species and only one $\mathrm{Ni}$ "ion. The spectrum was first simulated considering $S=1 / 2$ species with the following $g_{\text {eff }}$ values $g_{\text {xeff }}=2.3499, g_{\text {yeff }}=$ $2.2205, g_{\text {xeff }}=2.0293$ (Figure S5). The $g_{\text {eff }}$ values are consistent with resonances belonging to a $S=1 / 2$ species coming from an antiferromagnetic coupling between $S=1$ and $S=1 / 2$ species; the $q_{\text {eff }}$ values for an effective spin $S_{\text {eff }}=1 / 2$ due to a ferromagnetic coupling between a $S=1$ and a $S=1 / 2$ are completely different from those observed. ${ }^{23}$

It is possible, then, to use the relationship between $q_{\text {eff }}$ and the local $g_{\text {rad }}$ and $g_{N i}$ values $\left(g_{\text {eff }}=-g_{\text {rad }} / 3+4 g_{N i} / 3\right)$ to compute the $\underline{q}_{\mathrm{Ni}}$ components assuming $g_{\mathrm{raq}}=2.000$. We obtain $g_{\mathrm{XNi}}=2.022$, $q_{y N i}=2.1650$ and $g_{z N i}=2.2622$ with $g_{a v N i}=2.15$ consistent for $a$ $\mathrm{Ni}(\mathrm{II})$ square pyramidal geometry. ${ }^{24}$ The final step consisted in simulating the experimental spectrum by fixing the $g_{i N i}$ values, fixing a negative value for $J$ and changing the $D_{N i}$ value. The best calculated spectrum gives a positive $D_{N i}$ value and a $|J| / D=20$ (the spectra depend only on $|J| / D$ ). Negative $D$ values fail to reproduce the exact resonances for any $J / D$ ratio. This analysis leads to the conclusion that in the two-reduced species, the central ligand electronic density is strongly antiferromagnetically coupled to one of the $\mathrm{Ni}(\mathrm{II})$ ions possessing the distorted square pyramidal geometry. Since the $D$ value for pseudo-spy Ni(II) geometry is larger than $20 \mathrm{~cm}^{-1}$, the exchange parameter coupling is, therefore, larger (in absolute value) than $400 \mathrm{~cm}^{-1}$ consistent with the literature. ${ }^{20}$

The fit of the spectrum leads to the following values: $g_{\text {rad }}=$ $2.000, g_{x N i}=2.022, g_{y N i}=2.154, g_{z N i}=2.265 ; \mathrm{J}_{\mathrm{radNi}}$ and $\nabla_{N i}$ were set to $=200$ and $20 \mathrm{~cm}^{-1}$-respectively. The spectra are independent from the ratio $J / D$, and the relative intensities of the bands are better reproduced with positive $D$ values. ${ }^{22}$ The $g$ values are typical for $\mathrm{Ni}^{H}$-complexes with distorted geometry. 


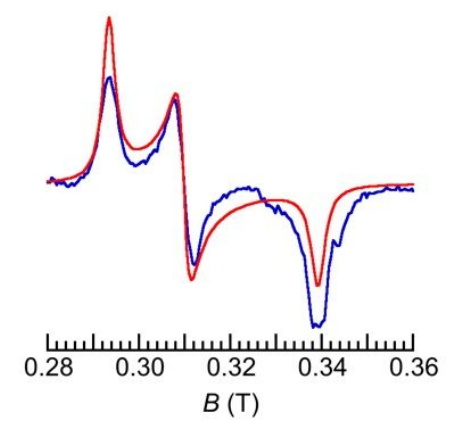

Figure 4. EPR spectra of two-electron reduced $\mathbf{1}$ in dichloromethane solution (blue) and the calculated spectrum (red) with the parameters in the text (microwave power $=1$ $\mathrm{mW}$, modulation amplitude $=8$ Gauss, gain $=50 \mathrm{db}$, temperature $=30 \mathrm{~K}$ ).

In conclusion, the electronic density of the trissemiquinone central ligand is relatively strongly antiferromagnetically coupled to the pseudo-tbp $\mathrm{Ni}(\mathrm{II})$ (Ni1) moiety and weakly coupled to the other two $\mathrm{Ni}(\mathrm{II})$ in the pristine species, while in the two-reduced species, it is very strongly coupled to one of the pseudo-spy $\mathrm{Ni}(\mathrm{II})$ ( $\mathrm{Ni2}$ or $\mathrm{Ni3}$ ) ions evidencing an electron transfer from one side of the molecule to another upon reduction. - W have evidenced that $\mathrm{Ni}_{3} \mathrm{HHTP}$ can be reversibly switched between different oxidation states of distinct magnetic properties that can, eventually, be addressed by an electrical field; evidencing, therefore, their potential to be used for the design of quantum devices._-HHTP and related bridging ligands containing nitrogen and sulfur instead of oxygen have been used during the last five years for the development of original two-dimensional (2D) coordination networks with paramagnetic ions presenting unique conducting properties and that can be used as sensors and/or for catalysis. ${ }^{25-31}$ Beyond the interest in electrically switchable discrete magnetic molecular complexes, investigating their electronic behavior may bring new insights and help understanding the synergy between the magnetic and the conducting properties of the $2 \mathrm{D}$ extended networks.

\section{Acknowledgement}

This work was supported by the Centre National de la Recherche Scientifique (CNRS, France) and Université Paris Sud. YW thanks the China Scholarship Council and Université ParisSud for financial support (No. 201506310023). TM thanks the Institut Universitaire de France for financial support.

\section{Conflicts of interest}

There are no conflicts to declare.

\section{Notes and references}

1 J. Lehmann, A. Gaita-Arin̄o, E. Coronado and D. Loss, Nat. Nanotech., 2007, 2, 312.
2 J. Ferrando-Soria, E. Moreno Pineda, A. Chiesa, A. Fernandez, S. A. Magee, S. Carretta, P. Santini, I. J. Vitorica-Yrezabal, F. Tuna, G. A. Timco, E. J. L. McInnes and R. E. P. Winpenny, Nat. Comm., 2016, 7, 11377.

3 X. Ma, E. A. Suturina, S. De, P. Négrier, M. Rouzières, R. Clérac and P. Dechambenoit, Angew. Chem. Int. Ed., 2018, 57, 78417845.

4 X. Z. Ma, E. A. Suturina, M. Rouzieres, M. Platunov, F. Wilhelm, A. Rogalev, R. Clerac and P. Dechambenoit, J. Am. Chem. Soc., 2019, 141, 7721-7725.

5 A. M. Barthram, R. L. Cleary, R. Kowallick and M. D. Ward, Chem. Commun., 1998, DOI: 10.1039/A807835A, 2695-2696.

6 C. S. Grange, A. J. H. M. Meijer and M. D. Ward, Dalton Trans., 2010, 39, 200-211.

7 Y. Suenaga, H. Inada, M. Inomata, R. Yamaguchi, T. Okubo, M. Maekawa and T. Kuroda-Sowa, Chem. Lett., 2014, 43, 562-564.

8 L. Yang, X. He and M. Dincă, J. Am. Chem. Soc., 2019, DOI: 10.1021/jacs.9b04822.

9 T. Deb, C. M. Anderson, H. Ma, J. L. Petersen, V. G. Young Jr and M. P. Jensen, Eur. J. Inorg. Chem., 2015, 2015, 458-467.

10 M. W. Lynch, R. M. Buchanan, C. G. Pierpont and D. N. Hendrickson, Inorg. Chem., 1981, 20, 1038-1046.

11 C. G. Pierpont and R. M. Buchanan, Coord. Chem. Rev., 1981, 38 , 45-87.

12 C. Benelli, A. Dei, D. Gatteschi and L. Pardi, Inorg. Chem., 1988, 27, 2831-2836.

13 A. Caneschi, A. Dei, H. Lee, D. A. Shultz and L. Sorace, Inorg. Chem., 2001, 40, 408-411.

14 A. Caneschi, A. Dei, C. P. Mussari, D. A. Shultz, L. Sorace and K. E. Vostrikova, Inorg. Chem., 2002, 41, 1086-1092.

15 S. Gomez-Coca, E. Cremades, N. Aliaga-Alcalde and E. Ruiz, J. Am. Chem. Soc., 2013, 135, 7010-7018.

16 R. Ruamps, L. J. Batchelor, R. Maurice, N. Gogoi, P. JimenezLozano, N. Guihery, C. de Graaf, A. L. Barra, J. P. Sutter and T. Mallah, Chem. Eur. J., 2013, 19, 950-956.

17 R. Ruamps, R. Maurice, L. Batchelor, M. Boggio-Pasqua, R. Guillot, A. L. Barra, J. Liu, E. E. Bendeif, S. Pillet, S. Hill, T. Mallah and N. Guihéry, J. Am. Chem. Soc., 2013, 135, 3017-3026.

18 B. Cahier, M. Perfetti, G. Zakhia, D. Naoufal, F. El-Khatib, R. Guillot, E. Rivière, R. Sessoli, A.-L. Barra, N. Guihéry and T. Mallah, Chem. Eur. J., 2017, 23, 3648-3657.

19 N. F. Chilton, R. P. Anderson, L. D. Turner, A. Soncini and K. S. Murray, J. Comput. Chem., 2013, 34, 1164-1175.

20 C. Cox, D. Isaacs, M. A. Bezpalko, W. S. Kassel, M. T. KieberEmmons and W. G. Dougherty, Polyhedron, 2019, 162, 165-170.

21 D. A. Shultz, K. E. Vostrikova, S. H. Bodnar, H.-J. Koo, M.-H. Whangbo, M. L. Kirk, E. C. Depperman and J. W. Kampf, J. Am. Chem. Soc., 2003, 125, 1607-1617.

22 C. Benelli, A. Dei, D. Gatteschi and L. Pardi, Inorg. Chem., 1990, 29, 3409-3415.

23 A. Bencini and D. Gatteschi, EPR of exchanged coupld systems, Springer-Verlag, New York, Berli, Heidelberg, 1990.

24 C. Benelli, A. Dei, D. Gatteschi and L. Pardi, Inorg. Chem., 1989, 28, 1476-1480.

25 M. Hmadeh, Z. Lu, Z. Liu, F. Gándara, H. Furukawa, S. Wan, V. Augustyn, R. Chang, L. Liao, F. Zhou, E. Perre, V. Ozolins, K. Suenaga, X. Duan, B. Dunn, Y. Yamamto, O. Terasaki and O. M. Yaghi, Chem. Mater., 2012, 24, 3511-3513.

26 M. G. Campbell, S. F. Liu, T. M. Swager and M. Dincă, J. Am. Chem. Soc., 2015, 137, 13780-13783.

27 H. Maeda, R. Sakamoto and H. Nishihara, Langmuir, 2016, 32 2527-2538. 
28 J. A. DeGayner, I.-R. Jeon, L. Sun, M. Dincă and T. D. Harris, J. Am. Chem. Soc., 2017, 139, 4175-4184.

29 X.-H. Liu, W.-L. Hu, W.-J. Jiang, Y.-W. Yang, S. Niu, B. Sun, J. Wu and J.-S. Hu, Appl. Mater. Interf., 2017, 9, 28473-28477.

30 M.-S. Yao, X.-J. Lv, Z.-H. Fu, W.-H. Li, W.-H. Deng, G.-D. Wu and G. Xu, Angew. Chem. Int. Ed., 2017, 56, 16510-16514.

31 V. Rubio-Giménez, M. Galbiati, J. Castells-Gil, N. Almora-Barrios, J. Navarro-Sánchez, G. Escorcia-Ariza, M. Mattera, T. Arnold, J. Rawle, S. Tatay, E. Coronado and C. Martí-Gastaldo, Adv. Mater., 2018, 30, 1704291. 
Supporting Information

Electronic and spin delocalization in a switchable trinuclear triphenylene trisemiquinone bridged $\mathrm{Ni}_{3}$ complex

Yiting Wang ${ }^{1}$, François Lambert ${ }^{1}$, Eric Rivière ${ }^{1}$, Régis Guillot ${ }^{1}$, Christian Herrero $^{1}$, Antoine Tissot $^{2}$, Zakaria Halime ${ }^{1}$, Talal Mallah ${ }^{*}$

${ }^{1}$ Institut de Chimie Moléculaire et des Matériaux d'Orsay, Université Paris-Sud, CNRS, Université Paris-Saclay, 91405 Orsay cedex, France

${ }^{2}$ Institut des Matériaux Poreux de Paris, UMR 8004 CNRS, École Normale Supérieure, Ecole Supérieure de Physique et de Chimie Industrielles de Paris, PSL University, 75005 Paris, France

Keywords: triphenylene, trisemiquinone, switchable, Ni, HHTP

\section{I-Synthesis and characterization}

$\mathrm{KBH}\left(\mathrm{TP}^{\mathrm{Ph}}, \mathrm{Ph}\right)_{3}(1.89 \mathrm{~g}, 2.7 \mathrm{mmol})$ was dissolved in $60 \mathrm{~mL} \mathrm{CHCl}{ }_{3}$, and the colorless solution was added into a methanolic solution of $\mathrm{NiCl}_{2} \cdot 6 \mathrm{H}_{2} \mathrm{O}(0.71 \mathrm{~g}, 3 \mathrm{mmol})$ with stirring. A pink precipitate was generated immediately. Two hours later, the solid was filtered, washed with a small amount of $\mathrm{CHCl}_{3}$ and dried under vacuum. The pink solid obtained corresponds to $\mathrm{Ni}\left[\mathrm{BH}\left(\mathrm{TP}^{\mathrm{Ph}}, \mathrm{Ph}\right)_{3}\right] \mathrm{Cl}$.

HHTP (32.5 mg, $0.01 \mathrm{mmol}$ ) was dissolved in a methanolic solution (15 mL) containing $0.6 \mathrm{~mL}$ of tetrabutylammonium hydroxide (TBAOH). The obtained dark violet solution was added to a $15 \mathrm{~mL}$ $\mathrm{CH}_{2} \mathrm{Cl}_{2}$ solution of $\mathrm{Ni}\left[\mathrm{BH}\left(\mathrm{TPPh}^{\mathrm{Ph}}\right)_{3}\right] \mathrm{Cl}(229 \mathrm{mg}, 0.3 \mathrm{mmol})$ with stirring. A darkish blue suspension was produced. The reaction was left overnight, and then filtered. A black precipitate was obtained, it was thoroughly washed with methanol. Yield $62 \%, 156 \mathrm{mg}$. The solid was redissolved in $\mathrm{CHCl}_{3}$, and the final solution was obtained after chromatography $\left(\mathrm{SiO}_{2}\right)$. The solvent was completely removed using a rotatory evaporator. The solid obtained was dissolved in acetone and crystals suitable for $X$ ray analysis were obtained by slowly evaporating acetone. Elem Anal. for $\mathrm{Ni}_{3}(\mathrm{HHTP})\left[\mathrm{BH}\left(\mathrm{TP}^{\mathrm{Ph}, \mathrm{Ph}}\right)_{3}\right]_{3}\left(\mathrm{C}_{3} \mathrm{H}_{6} \mathrm{O}\right)_{0.5}$ Calcd: C, 73.19\%; H, 4.53\%; N, 9.94\%; Found: C, 73.14\%; H, 4.35\%; $\mathrm{N}, 10.04 \% . \mathrm{M}_{\mathrm{W}}=2535.19 \mathrm{~g} / \mathrm{mol}$. Single-crystal unit cell: Triclinic, space group, $P-1 . \mathrm{a}=14.2552(18)$ $\AA, b=17.287(2) \AA, c=32.407(4) \AA, \alpha=94.408(4)^{\circ}, \beta=101.051(4)^{\circ}, \gamma=101.473(4)^{\circ}, V=7626.4(17)$ $\AA^{3}$. IR (KBr) v/cm-1: 3456 (w), 3062 (w), 2617 (w), 1603 (w), 1545 (s), 1502 (s), 1479 (s), 1462 (s), $1364(\mathrm{~m}), 1329(\mathrm{~m}), 1282(\mathrm{w}), 1232(\mathrm{w}), 1170(\mathrm{~m}), 1118(\mathrm{w}), 1064(\mathrm{~m}), 1013(\mathrm{~m}), 972(\mathrm{w}), 915(\mathrm{w})$, $844(w), 827(w), 803(s), 759(s), 696(s), 669(m), 619(w), 571(m), 543(m), 403(w), 353(w)$. 


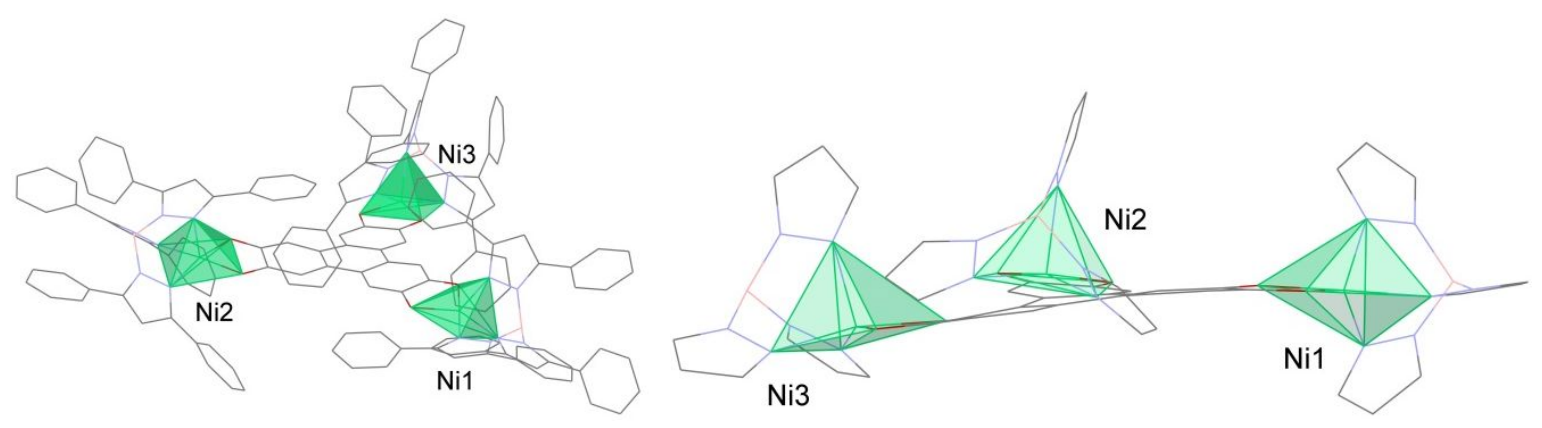

Figure S1. Polyhedral representation of the crystal structure

X-ray diffraction data for compound 1 was collected by using a VENTURE PHOTON100 CMOS Bruker diffractometer with Micro-focus luS source Mo K $\alpha$ radiation. Crystal was mounted on a CryoLoop (Hampton Research) with Paratone-N (Hampton Research) as cryoprotectant and then flashfrozen in a nitrogen-gas stream at $100 \mathrm{~K}$. The temperature of the crystal was maintained at the selected value by means of an $\mathrm{N}$-Helix to within an accuracy of $\pm 1 \mathrm{~K}$. The data were corrected for Lorentz polarization, and absorption effects. The structures were solved by direct methods using SHELXS$97^{1}$ and refined against $F^{2}$ by full-matrix least-squares techniques using SHELXL-2018 ${ }^{2}$ with anisotropic displacement parameters for all non-hydrogen atoms. All calculations were performed by using the Crystal Structure crystallographic software package WINGX. ${ }^{3}$

The crystal data collection and refinement parameters are given in Table S1.

CCDC 1936818 contains the supplementary crystallographic data for this paper. These data can be obtained free of charge from the Cambridge Crystallographic Data Centre via http://www.ccdc.cam.ac.uk/Community/Requestastructure.

Table S1. Crystallographic data and structure refinement details.

\begin{tabular}{|c|c|}
\hline Compound & 1 \\
\hline CCDC & 1936818 \\
\hline Empirical Formula & $\mathrm{C}_{153} \mathrm{H}_{108} \mathrm{~B}_{3} \mathrm{~N}_{18} \mathrm{Ni}_{3} \mathrm{O}_{6}, 7\left(\mathrm{C}_{3} \mathrm{H}_{6} \mathrm{O}\right)$ \\
\hline$M_{r}$ & 2909.67 \\
\hline Crystal color & black \\
\hline Crystal size, $\mathrm{mm}^{3}$ & $0.100 \times 0.095 \times 0.035$ \\
\hline Crystal system & triclinic \\
\hline Space group & $P-1$ \\
\hline $\mathrm{a}, \AA$ & $14.2552(18)$ \\
\hline b, $\AA$ & $17.287(2)$ \\
\hline c, $\AA$ & $32.407(4)$ \\
\hline
\end{tabular}

\footnotetext{
${ }^{1}$ Sheldrick, G. M. SHELXS-97, Program for Crystal Structure Solution, University of Göttingen, Göttingen, Germany, 1997.

${ }^{2}$ Sheldrick, G. M. Acta Crystallogr., Sect. A: Found. Crystallogr., 2008, 64, 112-122.

${ }^{3}$ Farrugia, L. J. J. Appl. Cryst. 1999, 32, 837.
} 


\begin{tabular}{|c|c|}
\hline$\alpha,{ }^{\circ}$ & $94.408(4)$ \\
\hline$\beta,{ }^{\circ}$ & 101.051(4) \\
\hline$v^{\circ}$ & $101.473(4)$ \\
\hline Cell volume, $\AA^{3}$ & $7626.4(17)$ \\
\hline$Z ; Z^{\prime}$ & $2 ; 1$ \\
\hline $\mathrm{T}, \mathrm{K}$ & $100(1)$ \\
\hline $\begin{array}{c}\text { Radiation type ; } \\
\text { wavelength } \AA\end{array}$ & MoK $\alpha ; 0.71073$ \\
\hline $\mathrm{F}_{000}$ & 3046 \\
\hline$\mu, \mathrm{mm}^{-1}$ & 0.435 \\
\hline$\theta$ range, $^{\circ}$ & $2.156-30.951$ \\
\hline Reflection collected & 217860 \\
\hline Reflections unique & 47603 \\
\hline $\mathrm{R}_{\text {int }}$ & 0.3232 \\
\hline GOF & 1.015 \\
\hline Refl. obs. $(I>2 \sigma(I))$ & 16901 \\
\hline Parameters & 1856 \\
\hline$w_{2}$ (all data) & 0.3393 \\
\hline $\mathrm{R}$ value $(I>2 \sigma(I))$ & 0.1390 \\
\hline $\begin{array}{c}\text { Largest diff. peak and } \\
\text { hole }\left(\mathrm{e}-. \AA^{-3}\right)\end{array}$ & $1.483 ;-1.004$ \\
\hline
\end{tabular}

Table S2. Some selected bonds distances and angles of complex

\begin{tabular}{|c|c|c|c|c|c|}
\hline \multicolumn{6}{|l|}{ Complex } \\
\hline \multicolumn{2}{|l|}{$\mathrm{Ni1}$} & \multicolumn{2}{|l|}{$\mathrm{Ni} 2$} & \multicolumn{2}{|l|}{$\mathrm{Ni3}$} \\
\hline $\mathrm{d}_{\mathrm{Ni1}-\mathrm{N} 1}$ & $2.009(5)$ & $\mathrm{d}_{\mathrm{Ni2}-\mathrm{N} 7}$ & $2.057(6)$ & $d_{\mathrm{Ni3-N13}}$ & $2.038(5)$ \\
\hline $\mathrm{d}_{\mathrm{Ni1}-\mathrm{N} 2}$ & $1.987(5)$ & $\mathrm{d}_{\mathrm{Ni2}-\mathrm{N} 8}$ & $2.031(7)$ & $d_{\mathrm{Ni3}-\mathrm{N} 14}$ & $2.018(5)$ \\
\hline$d_{\text {Ni1-N3 }}$ & $2.067(5)$ & $\mathrm{d}_{\mathrm{Ni2-N9}}$ & $2.040(7)$ & $d_{\text {Ni3-N15 }}$ & $2.028(5)$ \\
\hline$d_{\text {Ni1-01 }}$ & $2.039(4)$ & $d_{\mathrm{Ni2}-03}$ & $1.984(6)$ & $d_{\mathrm{Ni3-05}}$ & $1.971(4)$ \\
\hline$d_{\text {Ni1-02 }}$ & $1.957(5)$ & $d_{\mathrm{Ni2}-04}$ & $2.020(5)$ & $d_{\mathrm{Ni3-06}}$ & $2.022(4)$ \\
\hline$d_{C 3-01}$ & $1.247(7)$ & $d_{C 9-03}$ & $1.293(9)$ & $\mathrm{d}_{\mathrm{C} 15-05}$ & $1.291(7)$ \\
\hline $\mathrm{d}_{\mathrm{C4-02}}$ & $1.300(7)$ & $\mathrm{d}_{\mathrm{C} 10-04}$ & $1.270(8)$ & $\mathrm{d}_{\mathrm{C} 16-06}$ & $1.278(7)$ \\
\hline $\mathrm{d}_{\mathrm{C3}-\mathrm{C} 4}$ & $1.469(8)$ & $\mathrm{d}_{\mathrm{C} 9-\mathrm{C} 10}$ & $1.459(10)$ & $d_{C 15-C 16}$ & $1.448(9)$ \\
\hline $01 \widehat{\mathrm{Ni} 10} 2$ & $81.94(17)$ & $03 \widehat{\mathrm{Ni} 2 \mathrm{O}} 4$ & $81.8(2)$ & $05 \widehat{\mathrm{Ni} 306}$ & $81.92(17)$ \\
\hline
\end{tabular}




\section{$\underline{\text { III-Electrochemical Characterization Procedures }}$}

Cyclic voltammetry measurements were performed in an electrochemical cell composed of a glassy carbon ( $3 \mathrm{~mm}$ diameter) working electrode, a saturated calomel electrode (SCE) as a reference electrode and a platinum mesh counter electrode. Dichloromethane (Fisher Chemical 99+\%) was distilled over $\mathrm{CaCl}_{2}$ and tetra- $N$-butylammonium hexafluorophosphate ( $\left[\mathrm{Bu}_{4} \mathrm{~N}\right] \mathrm{PF}_{6}$, Aldrich $99 \%$ ) was recrystallized in ethanol. Solutions of the complex were prepared at a concentration of $1 \mathrm{mM}$ and $\left[\mathrm{Bu}_{4} \mathrm{~N}\right] \mathrm{PF}_{6}$ was used as supporting electrolyte and its concentration was maintained at a hundredfold excess compared to the sample. The solutions were purged with inert argon gas and the cyclic voltammograms were measured at a scan rate of $100 \mathrm{mV} / \mathrm{s}$.

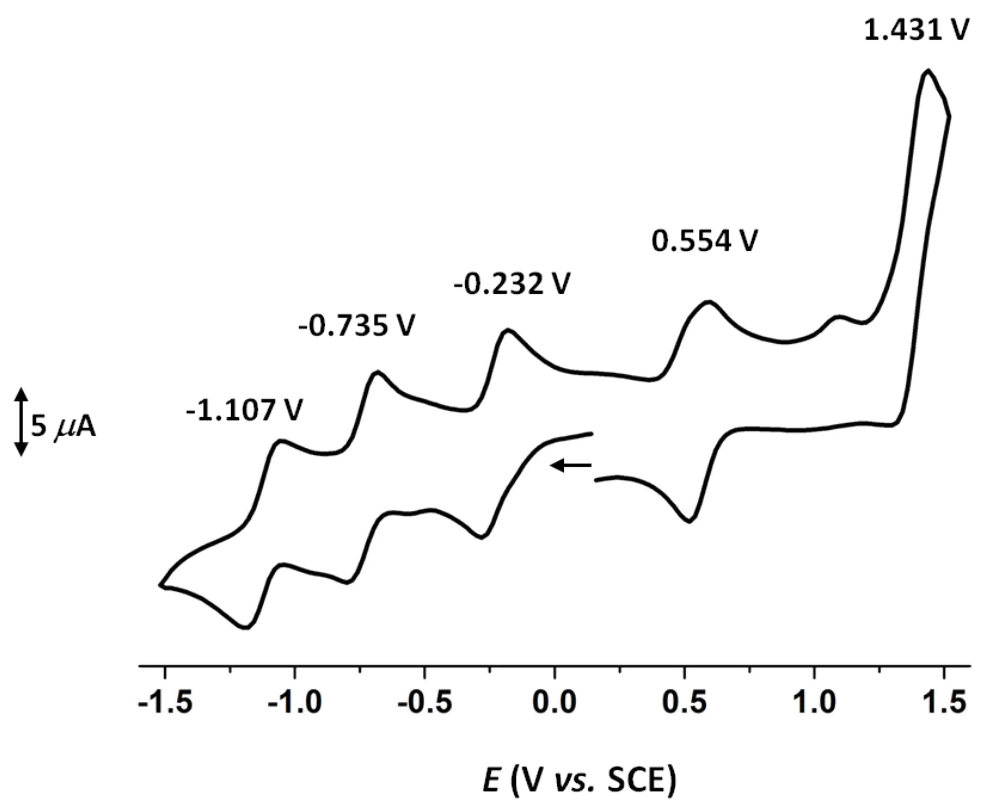

Figure S2. Cyclic voltammograms of $1 \mathrm{mM} 1$ in argon-degassed dichloromethane containing $0.1 \mathrm{M}$ $\left[\mathrm{Bu}_{4} \mathrm{~N}\right] \mathrm{PF}_{6}$ at $25{ }^{\circ} \mathrm{C}$ and recorded using a glassy carbon working electrode, a platinum mesh as the counter electrode and a saturated calomel electrode (SCE) as the reference electrode.

Bulk electrolysis for the preparation of the samples for EPR studies was performed in the same conditions as for cyclic voltammetry experiments except for the glassy carbon working electrode that was replaced by $5 \mathrm{~mm}^{3}$ carbon foam with high effective surface (Alfa Aesar. carbon felt 99.0\%). After each electrolysis, samples were transferred using a cannula under argon atmosphere into EPR tubes and then frozen immediately in a liquid nitrogen bath. 


\section{IV-Magnetic studies}

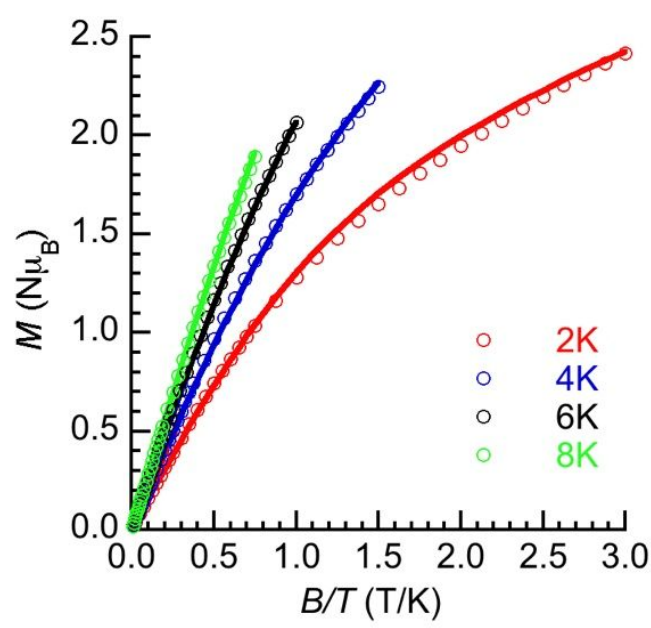

Figure $\mathrm{S3} . \mathrm{M}=\mathrm{f}(B / T)$ for 1 .

The magnetic data were fitted by fixing the rhombic parameters $E$ for the three $\mathrm{Ni}^{\prime \prime}$ to zero. Letting the $E$ values free during the fitting procedure does not lead to a large change in the axial ZFS parameters $D$; the $J$ values are also not very different. The best fit solution is not unique. Other solutions can be found by interchanging the $J$ values; they however remain in the same range: one strongly antiferromagnetic, one weakly antiferromagnetic and weakly ferromagnetic. Furthermore, one solution that have a good agreement with the magnetic experimental data with one very strong antiferromagnetic coupling $\left(-800 \mathrm{~cm}^{-1}\right)$ and two extremely weak coupling (few $\left.\mathrm{cm}^{-1}\right)$ was also found. However, this solution leads to ground state with $S=1 / 2$ very separated from the excited states, which does not correspond to the EPR data. This solution was discarded.

\section{$\underline{\text { V-Electron Paramagnetic Resonance }}$}

X-band EPR spectra were recorded on a Bruker ELEXSYS 500 spectrometer equipped with a Bruker ER 4116DM X band resonator, an Oxford Instrument continuous flow ESR 900 cryostat, and an Oxford ITC 503 temperature control system.



Figure S4. EPR spectra of 1 in dichloromethane solution (blue) and the solid state (red) at $\mathrm{T}=5 \mathrm{~K}$ 
(Microwave power $=1 \mathrm{~mW}$, modulation amplitude $=8$ Gauss, gain $=50 \mathrm{db}$, temperature $=5 \mathrm{~K}$ ).

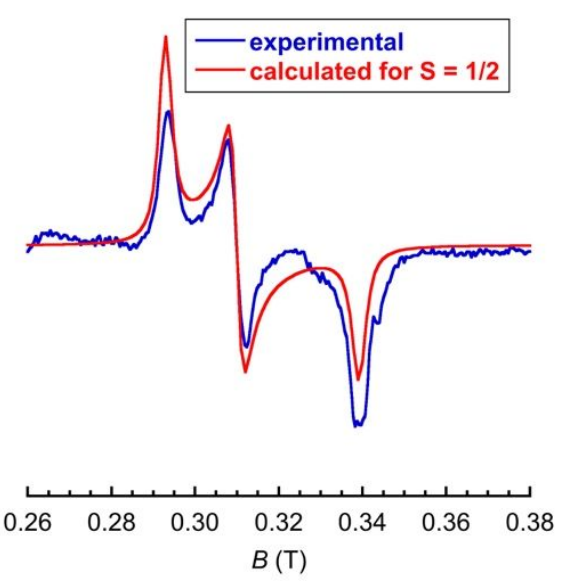

Figure S5. EPR spectra of the two-reduced species (blue) ((microwave power $=1 \mathrm{~mW}$, modulation

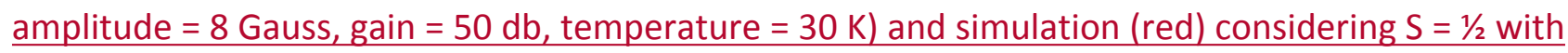
the following $g_{\text {eff }}$ values $g_{\text {xeff }}=2.3499, \mathrm{~g}_{\text {yeff }}=2.2205, \mathrm{~g}_{\text {xeff }}=2.0293$ 
Electronic and spin delocalization in a switchable trinuclear triphenylene trisemiquinone bridged $\mathrm{Ni}_{3}$ complex

Yiting Wang ${ }^{1}$, François Lambert ${ }^{1}$, Eric Rivière ${ }^{1}$, Régis Guillot ${ }^{1}$, Christian Herrero ${ }^{1}$, Antoine Tissot $^{2}$, Zakaria Halime ${ }^{1}$, Talal Mallah ${ }^{1 *}$

\section{Table of Contents}

An electro- switchable trinuclear $\mathrm{Ni}_{3}$-trisemquinone complex with electronic and spin dependent oxidation state delocalization
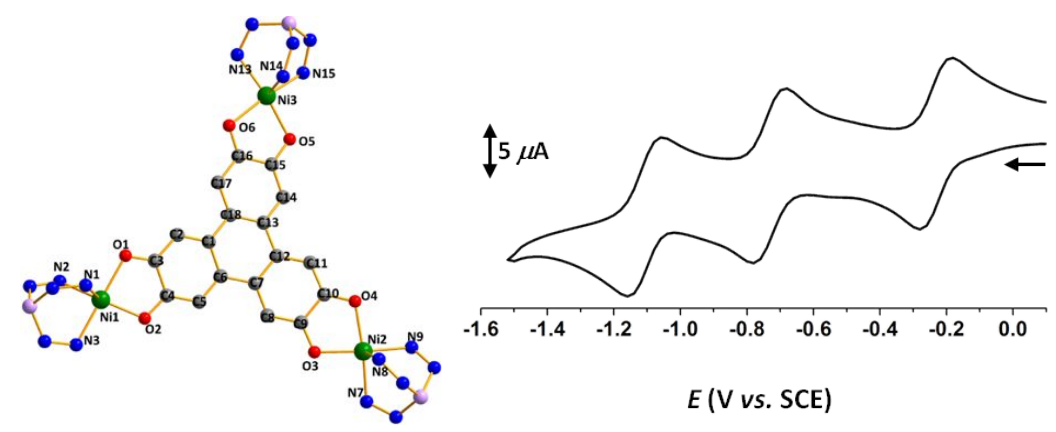